\title{
PMTR: Privacy-enhancing Multilayer Trajectory-based Routing Protocol for Vehicular ad hoc Networks
}

\author{
Baber Aslam ${ }^{\ddagger}$, Faisal Amjad ${ }^{\dagger}$ and Cliff C. Zou ${ }^{\dagger}$ \\ ${ }^{\ddagger}$ National University of Sciences and Technology, Islamabad, Pakistan ${ }^{\ddagger}$ \\ ${ }^{\dagger}$ University of Central Florida, Orlando, FL, USA \\ †baber-mcs@nust.edu.pk, ${ }^{\dagger}$ \{faisal, czou\}@cs.ucf.edu
}

\begin{abstract}
In this paper we present a trajectory-based routing protocol for vehicular ad hoc networks (VANETs) that routes messages using historical vehicular traffic statistics and coarse privacy-preserving trajectory information provided by vehicles. The protocol is well suited for the initial deployment stage of VANET when the number of VANET-enabled vehicles and roadside units will be very low. Coarse trajectory information preserves privacy of carrier vehicles by allowing vehicles only to report their intended rough travel regions instead of detailed travel paths, and historical vehicular traffic statistics ensure high success rate of message delivery. We have compared the proposed routing protocol with three other routing protocols: a flooding type protocol, a geographical or position-based protocol, where message is greedily forwarded towards destination location using carriers' destination information (GeoDest), and a geographical or position-based protocol using carriers' exact trajectory information (GeoTrace). Simulation results have shown that our proposed protocol performs better than GeoDest and equally well or better than GeoTrace (while GeoTrace does not protect carrier vehicles' privacy). Further, it has far less overheads as compared to Flooding and performs equally well as Flooding in one of the operating modes.
\end{abstract}

Keywords- vehicular networks; initial deployment stage; routing; trajectory; privacy

\section{INTRODUCTION}

Vehicular ad hoc networks (VANETs) are a special case of mobile ad hoc networks (MANETs), where the vehicles are the mobile nodes. The mobile nodes exhibit high mobility but the movement of these mobile nodes is no more random but restricted to roads and streets. When the density of VANETenabled vehicles is too small, vehicles to vehicles direct communication could be frequently disrupted. The dynamic and often disconnected network topology of VANET makes it quite inefficient to use standard MANET routing protocols (such as AODV [1]) in VANET applications [2].

The simplest VANET routing protocol is to use flooding (broadcast, epidemic etc) mechanism to route messages. This type of routing protocols does not incorporate any information about underlying VANET and could achieve a high success rate, but at the cost of high overheads. The second type of routing protocols incorporates the location of destination in routing a message and greedily forward the message towards the destination location, commonly referred as a geographical or position-based routing [3,4]. Although this type of routing protocols has less overhead compared to flooding type of protocols, it has lower success rate due to local maxima, where no path/route/road (that can reduce the distance of a message to its destination) exists between the current message location and its destination. The third type of routing protocols incorporates, in addition of location information, the topology of road network. It routes a message along roads using roadintersections as routing points instead of nodes [5] or use other recovery mechanisms such as perimeter mode in [6]. This type of routing protocols overcomes the local maxima problem due to non-existent path that is caused by non-availability of roads, but may fail due to disconnected nature of VANET that is caused by uneven distribution of vehicles along different roads [7]. The fourth type of routing protocols incorporates vehicle distribution (traffic data) to select the road segments that have high probability of having a connected vehicle-to-vehicle communication $(\mathrm{V} 2 \mathrm{~V})$ path $[8,9]$.

We are more interested in the initial deployment stage of VANET (in the near future) than the mature stage of VANET (in the far future). Because of the long lifetime of existing vehicles and the complexity of setting VANET (in the largescale and dynamic automobile system), the initial transition stage of VANET development is anticipated to last for a long time and is critical to the future of VANET. The initial deployment stage of VANET will be characterized by low market penetration of VANET-enabled vehicles (henceforth referred as vehicles) thus resulting in failure of $\mathrm{V} 2 \mathrm{~V}$ communication in most of the time. All the routing protocols that depend on V2V communication to route messages will, therefore, not work well during the initial deployment stage of VANET.

One possible solution, to the routing during initial deployment stage of VANET, is to make use of carry-andforward $(\mathrm{C} \& \mathrm{~F})$ paradigm, where a vehicle carries a message till it encounters a suitable next carrier [10]. The routing mechanisms discussed earlier (such as flooding, positionbased, position-based with road-topology) can also be used with C\&F mechanism but will have same disadvantages as explained earlier. The limited number of vehicles, during the initial deployment stage, makes it impossible to achieve a connected V2V path; therefore vehicles' trajectory information 
is usually utilized to forward a message towards its destination [11]. The trajectory-based mechanism can result in local maxima, when a message is routed to a place where the likelihood of finding the next carrier is very low.

Further, some protocols require vehicles to divulge exact trajectory information, which may be undesirable from user's privacy preferences [12]. Privacy is one of the most important security attributes of VANET and may make a solution unacceptable to users. Some protocols provide privacy by sharing message destination instead of vehicle trajectory/destination [11], but these solutions still suffer from local maxima.

In this paper we propose a routing protocol that successfully routes messages using historical vehicular traffic statistic information and a coarse trajectory information from users. The protocol can resolve the local maxima issue while at the same time preserve the privacy of vehicular users. In the proposed protocol, the road area is progressively divided into sub-regions in a layered manner (as illustrated in Fig. 2). Routing is done, at the top layer, based on historical vehicular traffic flows among these sub-regions or cells.

We have compared our proposed routing protocol with three other routing protocols: a flooding based protocol, a geographical or position-based protocol using vehicles' final destination information (GeoDest) and a geographical or position-based protocol using vehicles' exact trajectory information (GeoTrace). These three protocols have been defined/chosen to cover the range of dependence on information/privacy, i.e., flooding needs no information, GeoDest only needs destination information, and GeoTrace needs complete trace information. Simulation results have shown that our proposed protocol performs better than GeoDest and equally well or better than GeoTrace (while GeoTrace does not protect carrier vehicles privacy). Further, it has far less overheads as compared to Flooding and it performs equally well as Flooding in one of the operating modes.

The paper is organized in 6 sections. Section II discusses related research. Section III describes system. Section IV presents the proposed routing protocol. Section $\mathrm{V}$ gives simulation and its results. Section VI concludes the paper.

\section{RELATED WORK}

Rondinone et al. [13] use street maps and multi-hop connectivity to dynamically route the packets. At each intersection next routing path (road segment) is dynamically chosen based on multi-hop connectivity and progress towards destination. It may be difficult to get any multi-hop connectivity during initial deployment stages of VANET thus reducing the effectiveness of protocol. Further, as no traffic statistics are considered, it may also suffer from local maximum if the packet gets routed to a point from where the probability of finding a suitable carrier is very low.

Tian et al. [14] present a routing protocol that makes use of street map to find the shortest path; the packet is then forwarded along this path. If suitable next carrier is not available then the packet may be temporarily stored with current carrier. Since the protocol does not consider traffic statistics therefore the shortest path may not have sufficient vehicles to forward the packet.

Jerbi et al. [15] present a routing protocol that makes use of street map and real time traffic for routing. At each junction, next junction is selected based on traffic density between the junctions and location of destination. They employ carry and forward for recovery purposes. The availability of real time traffic distribution may be difficult especially during initial deployment stages of VANET, further it may also suffer from local maximum if the packet gets routed to a point from where the probability of finding a suitable carrier is very low.

Zhao et al. [16] use street maps and traffic statistics for routing the packet. They prefer transferring packets through connected road segments and carry the packets if no connected road segment is available; for carrying the packets higher speed road is preferred. Leontiadis et al. [11] use vehicle trajectory information to route packets closest to destination. Vehicle carries the packet till it reaches the destination of packet or finds a better carrier that can take the message closer to destination. Cheng et al. [7] define three modes for GeoDTN+Nav; greedy mode, perimeter mode and DTN mode. Packets are forwarded using greedy mode and if a local maximum is encountered then recovery is done using perimeter mode and if it fails then packet is carried by vehicles using DTN mode. It requires vehicles to report their navigation information thus effecting users' privacy. Although carry and forward mechanism in these protocols takes care of disconnected nature of VANET but packet delivery can still fail due to local maxima.

\section{SYSTEM MODEL}

The scope of this paper is restricted to the initial deployment stage of VANET that will be characterized by low market penetration of VANET-enabled vehicles and sparse deployment of roadside units (RSUs). Low market penetration of VANET-enabled vehicles will cause failure of applications/protocols that depend on $\mathrm{V} 2 \mathrm{~V}$ communication, whereas sparse RSU network will affect applications/protocols that depend on vehicle to roadside (V2R) communication. Our main focus, in this paper, is urban environment as shown in Fig. 1(a). The figure shows a partial map of Miami, FL, USA. The major roads are shown in color (blue, red and yellow) whereas smaller/local streets are shown in outline. Due to low market penetration, the number of VANET-enabled vehicles on small/local streets will be negligible therefore these small/local streets can be ignored; a simplified map is shown in Fig. 1(b).

It is assumed that vehicles are equipped with navigation system/GPS, have digital map (including cell divisions) of the area and are aware of the locations of roadside units (RSUs) in the area. We do not assume pervasive or fully networked RSUs. There can be few connected/networked RSUs and some 
standalone RSUs. A standalone RSU does not have an Internet access or network connection to other RSUs, but it can communicate with and be possibly controlled by a nearby vehicle via wireless networking using our protocol. It is also assumed that historical vehicular traffic statistics are available. These may be integrated with digital maps.

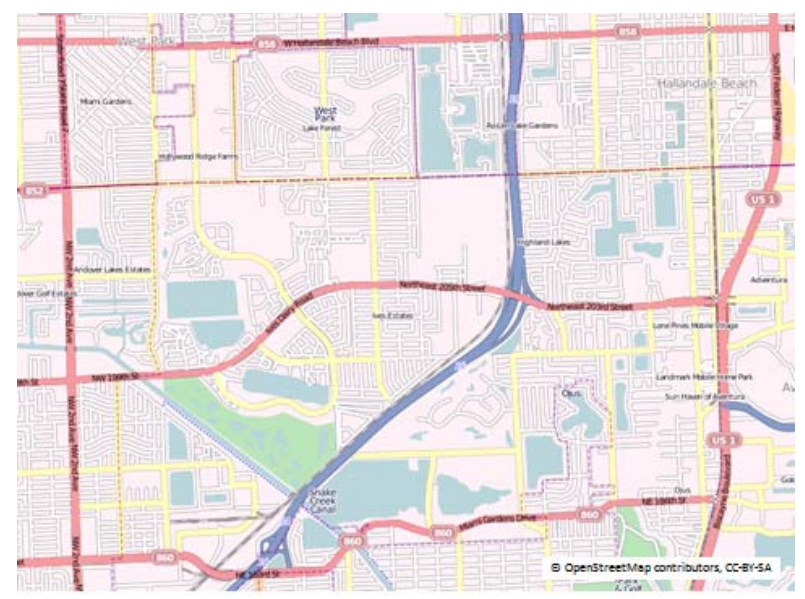

(a)

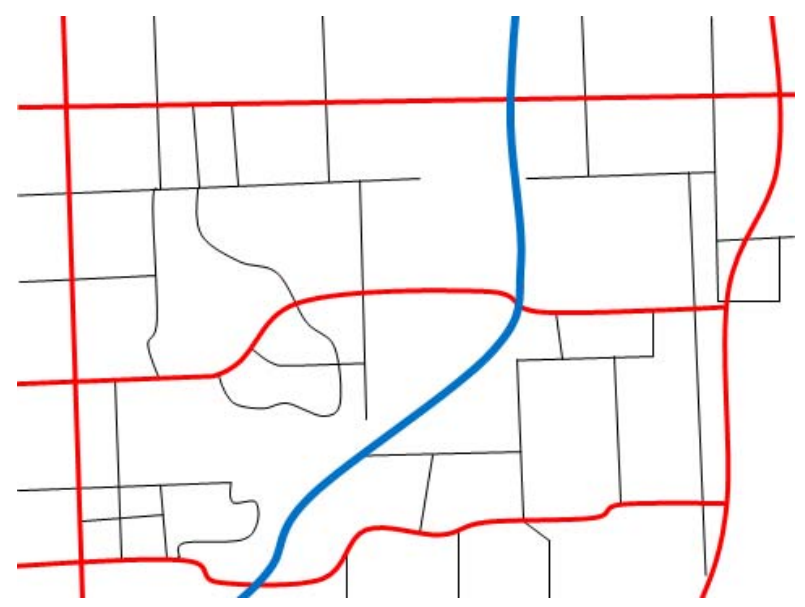

(b)

Figure 1. Urban Environment (a) Partial map of Miami, FL, USA (b) Simplified map of Figure 1(a).

\section{Proposed Routing Protocol}

\section{A. Layered division of area}

Area/region is divided into cells; refer Fig. 2 cells $A, B, C$ and $D$. Each cell is further divided into sub-cells (Fig. 2 cells $A 1, A 2, A 3$ and $A 4)$ and this layered sub-division continues (Fig. 2 cells $A 2 . I, A 2 . I I, A 2 . I I I$ and $A 2 . I V$ ) till the smallest cell size is reached. The size of the smallest cell will be defined by minimum privacy needs. The levels of sub-divisions are not fixed. The sizes of cells at any level are considered to be same but this is not a must. For example if we have a long section of a freeway with no diversion/ramps etc. then we keep the complete section of the freeway in one cell at the lowest level.

\section{B. Privacy Preservation based on Cell-level Trajecotry Routing}

The proposed routing protocol determines routing path based on cell-level trajectory information revealed by carrier vehicles. Cell-level trajectory means that a vehicle only reveals its trajectory in terms of what cells of area it will go through. In this way, the vehicle's user does not need to worry about exposing its detailed trajectory path and destination information to others. If a vehicle reports its trajectory in terms of higher-level cells, it will have stronger privacy protection, of course, at the cost of less efficiency in routing.

\section{Traffic Statistic}

Each cell has associated directional flows of traffic that are computed from historical vehicular traffic statistics. For two adjacent cells $A$ and $B$, the directional flow of traffic from cell $A$ to cell $B, D_{A B}$, (or from cell $B$ to cell $A, D_{B A}$ ) can be defined as the volume of traffic that is moving from cell $A$ to cell $B$ (or from cell $B$ to cell $A$ ). Note the computation is based on volume of traffic crossing the cell boundary and not on individual vehicle's traces. This makes data collection easy and also protects user privacy.

As the traffic patterns are not uniform throughout a day so traffic flows will also change with time. But at the same time, traffic patterns are repetitive, e.g., the traffic pattern between two points at a particular time of a work day will generally be same on all other work days of the week. Therefore, the historical traffic statistics can be used to compute traffic flows for a particular time period of a day.

\section{Proposed Routing Protocol}

PMTR is a source routing protocol where source specifies the route a message has to follow to reach the destination. The route is defined as the trajectory of cells that a message follows from source to destination. The selection of cells for a particular route is made in a manner to maximize the successful delivery rate, which is achieved by selecting cells such that the directional flow of traffic, from the source cell to the destination cell, is maximized.

The protocol uses $\mathrm{C} \& \mathrm{~F}$ mechanism where a message is carried by a vehicle until it finds the next suitable carrier. The vehicles provide trajectory information based on cells; the size of cell defines the privacy level. The forwarding/carrier vehicles are selected based on trajectory information provided by potential carrier vehicles and the routing trajectory of a message. The trajectory information can be included in periodic broadcast beacons. The selection of carrier is made to minimize the number of cells enroute destination. For example, if a particular route is defined as a trajectory of cells $A \rightarrow B \rightarrow E \rightarrow G \rightarrow H \rightarrow K$ and the trajectory of present carrier overlaps cell $E$ then the newly selected carrier must have an overlapping trajectory with cell $G$ or cell $H$ or cell $K$. 
Each cell is further divided into sub-cells. When a message reaches the destination-cell, it is routed further to the destination location based on sub-cells. The user is only required to provide sub-cell based trajectory information within the destination cell. If users elect not to provide this information then the message is broadcasted within the destination cell in order to finally reach the destination location. Thus the proposed routing protocol successfully routes the messages while enhancing the users' privacy.

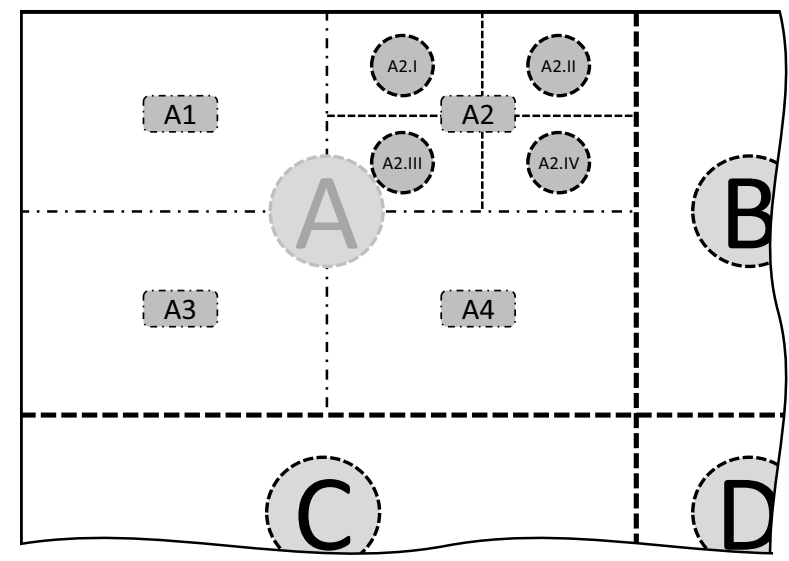

Figure 2. Layered division of area.

\section{Simulation}

\section{A. Simulation setup}

Simulation is based on urban area as shown in Fig. 3. Fig. 3(a) shows a partial map of Miami, FL, USA. During the initial deployment stages of VANET there will be very small number of VANET-enabled vehicles therefore the number of VANETenabled vehicles on small/local streets will be negligible. These small/local streets have, therefore, been omitted and the modified map shown in Fig. 1(b) is used for our simulation study. The region is $12 \mathrm{~km} \times 8 \mathrm{~km}$ with a total road length of $133.7 \mathrm{~km}$. The width/color of roads represent road classification in terms of speed and traffic volume; blue being the highest, followed by red and then black.

A total of 10,000 vehicle traces were generated for this simulation. The average speed of vehicles depends on the road classification. At any intersection, the probability of taking a road depends on the probability of turning and road classifications. We assume that the probability of taking a road is proportional to its road classification, i.e., the higher the road classification the larger value of the probability of taking that road. The probability of taking a turn is lower than the probability of no-turn. For each vehicle trace, a starting point on the map was randomly chosen; the vehicle then continues travelling till completion of trip with speed/direction as discussed above. The trip completes if one of the following condition is met a) vehicle moves off map b) vehicle intersects its own travelled path c) trip duration exceeds defined maximum value. If the trip duration of a generated vehicle trace is less than the defined minimum value then that vehicle trace is discarded.

For this simulation only one level of sub-divisions were considered. The cell size is $2 \mathrm{~km} \times 2 \mathrm{~km}$; resulting in a total of 24 cells. The volume directional flow of traffic among these cells in all four directions is calculated from 10,000 vehicle traces used for simulation. It is assumed that historic traffic statistics are available to all vehicles. Both source and destination are stationary; source is located in top-left cell and destination is located in bottom-right cell. A simplified forwarding strategy has been used; if no suitable carrier is found, before diversion or end of trip, the message is treated as lost and is not transferred to a standalone RSU or locally broadcasted in the cell. This simplification does not enhance the performance; PMTR may therefore perform better than the results of this simulation.

We have compared our proposed routing protocol with three different routing protocols covering a wide range of information from users: Flooding, GeoDest and GeoTrace. Flooding needs no trajectory information from users. As the name indicates, the source repeatedly broadcasts the message to every vehicle in range. All vehicles, in turn, repeatedly broadcast the message to every other vehicle in its transmission range. GeoDest is a geographical or position-based protocol which routes a message towards its destination location. It only requires potential carriers to report their destination information. The vehicle with its destination closest to the message destination is selected as the next carrier. GeoTrace is also a geographical or position-based protocol which routes the message towards its destination location. Unlike GeoDest approach, it requires the complete trajectory information from potential carriers. The vehicle whose trajectory passes closest to the message destination is selected as the next carrier.

We have defined different modes of operations for all the routing protocols (except for Flooding) named as S1V1, S5V1 and S5V5. The mode of operation defines the number of copies that will be generated for each message. "S $x \mathrm{~V} y$ " means the source forwards a message to $x$ different vehicles and each intermediate vehicle forwards the message to $y$ different vehicles. For example, "S5V1" means that the source passes its generated message to 5 carrier vehicles, and each of these carrier vehicles will carry and eventually forward the message to one another carrier vehicle for further delivery. Flooding uses only one mode of operation, $\mathrm{S} A L L \mathrm{~V} A L L$, the source repeatedly broadcasts the message to every vehicle in range. All vehicles, in turn, repeatedly broadcast the message to every other vehicle in its transmission range.

In order to check the performance of our protocol we have used 100 vehicles on the map or 1 vehicle $/ 1.33 \mathrm{~km}$. The quantity shows the number of VANET-enabled vehicles in our simulation area at any particular time. The simulation starts by picking given number of vehicles from generated-vehicletraces and placing them on the map, the vehicles then move on the map along their trajectory defined in generated-vehicletraces, whenever a vehicle leaves the map or finishes its trip a 
new vehicle is picked form the generated-vehicle-traces and is placed on the map. The process continues till the message reaches its destination.

\section{B. Performance Metrics used in Evaluation}

A total of 1000 unique messages are transmitted during each simulation run; depending on the routing protocol and mode of operation, multiple copies of a message may be generated. Statistics corresponding to the successful delivery of the first copy of each message to the destination are recorded. Based on these statistics, we have defined several performance metrics: Probability of success, Probability (Delivery time $<\mathrm{t}$ ) and Total number of hops. Probability of Success is the probability that a given message will be successfully delivered to its destination, which is computed from the number of unique messages successfully delivered to the destination. Probability (Delivery time $<t$ ) is the probability that the delivery time of a message is less than $t$, which is computed from the delivery time of each unique message. Total number of hops is a measure of total number of hops for all copies of a message. It is computed by averaging over all successfullydelivered-unique-messages. Total number of hops also shows the average number of carrier vehicles involved in delivery of message and is being used to measure the overhead.

\section{Results and discussion}

Fig. 3, 4 and 5 show the results for 100 vehicles on the map which is 1 vehicle $/ 1.33 \mathrm{~km}$. For different modes of operations, Fig. 3 shows the probability that the delivery time of a message is less than a given time. Long delivery times, even for flooding, are due to disconnected nature of network caused by low vehicle density. We see that for all modes of operation PMTR performs equally well as GeoTrace and better than GeoDest. It also performs equally well to Flooding under S5V5 mode of operation, Fig. 3(c). Fig. 4 show the probability of successfully delivering a message for all protocols in different operation modes. We see that for all modes of operation PMTR performs equally well as GeoTrace and better than GeoDest. Further, its performance matches that of Flooding in S5V5 mode of operation. Fig. 5 shows the total number of hops for all protocols in different operation modes. GeoDest has least number of hops, followed by GeoTrace, then PMTR and finally Flooding has maximum number of hops.

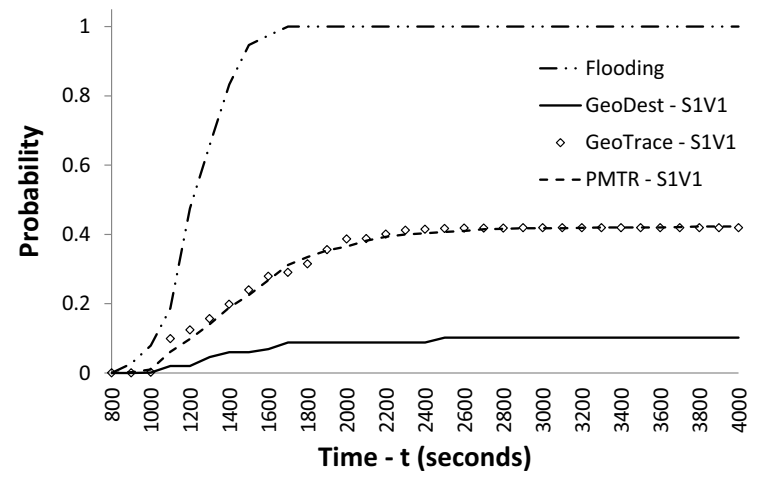

(a)

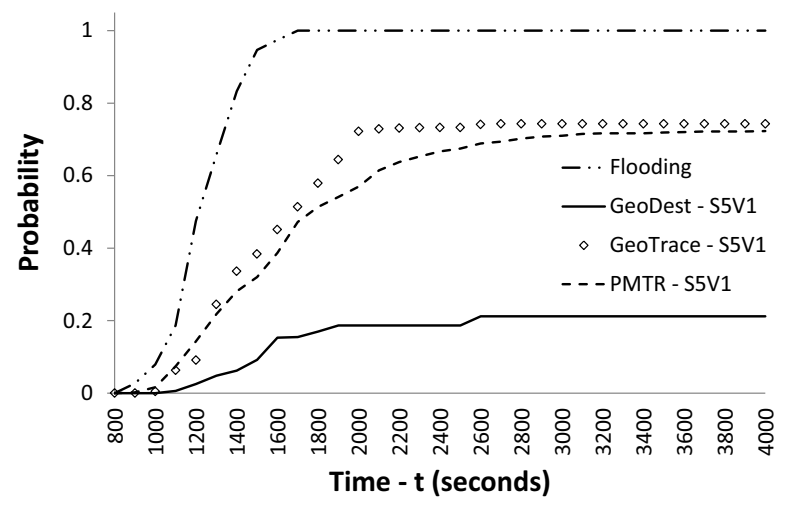

(b)

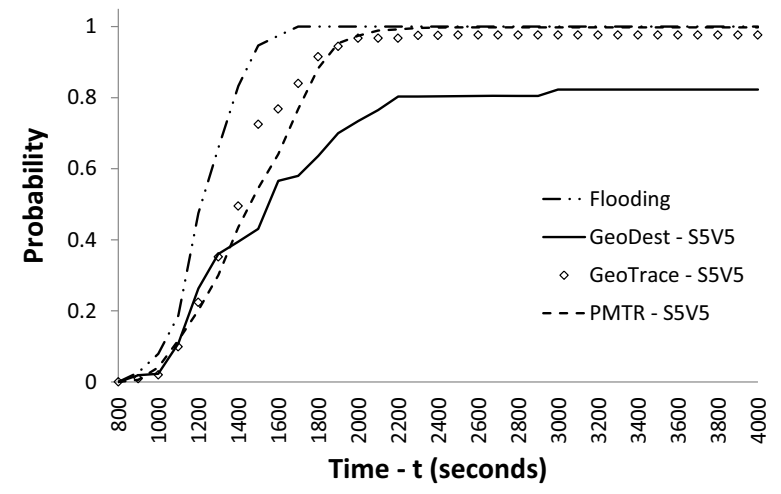

(c)

Figure 3. Probability (Delivery time $<t$ ): Probability that the delivery time of a message is less than a given time $t$ for 100 vehicles on map (i.e., 1 vehicle $/ 1.33 \mathrm{~km}$ ) for different modes of operation, (a) S1V1 (b) S5V1 (c) S5V5. Note- Flooding uses SALLVALL.

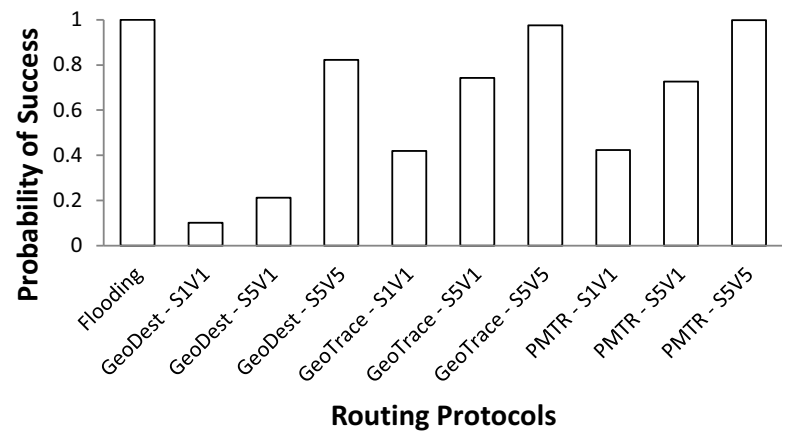

Figure 4. Probability of Success: Probability that a given message will be successfully delivered to its destination for 100 vehicles on map (i.e., 1 vehicle $/ 1.33 \mathrm{~km}$ ) for different modes of operation. 


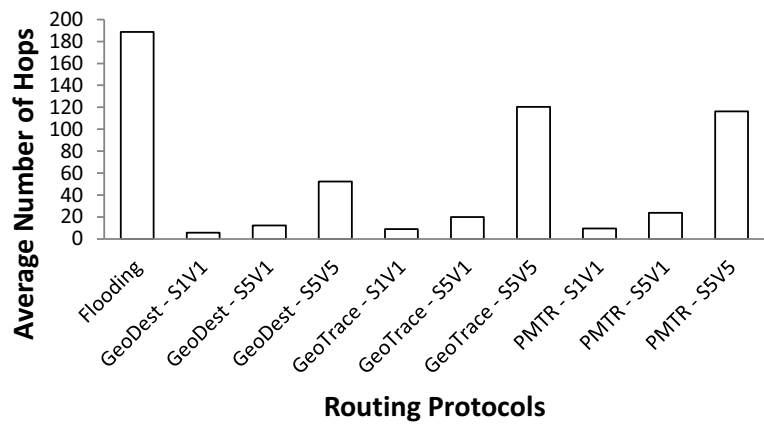

Figure 5. Total number of hops: Total number of hops for all copies of messages (i.e., total number of vehicles involved in the delivery of a message)

for 100 vehicles on map (i.e., 1vehicle $/ 1.33 \mathrm{~km}$ ) for different modes of operation.

Flooding provides best privacy and also performs best in terms of delivery time. However, it has a very high overhead cost in terms of total hops for all copies of messages, i.e., total number of vehicles involved in the delivery of messages. GeoDest provides the next lower-level of privacy but performs worst in all modes of operation; its overheads, however, are the smallest in each mode of operation. GeoTrace provides worst privacy but performs better than GeoDest in all modes of operation. Its overheads are higher than that of GeoDest. PMTR provides intermediate privacy, which is better than that of GeoTrace and perform better than GeoDest for all modes of operation. It performs equally well or better than GeoTrace while providing better privacy than GeoTrace. It also performs equally well as Flooding under S5V5 mode of operation with far less overheads, i.e., total number of hops for all copies of messages (which also indicates total number of vehicles involved in the delivery of message).

\section{CONCLUSION}

We have presented a routing protocol that is well suited during the initial deployment stage of VANET where the number of (smart) vehicles as well as the number of roadside infrastructure will be limited. Our routing protocol makes use of historical vehicle traffic statistics and coarse trajectory information form vehicle to route the messages. We have compared our protocol with three different protocols, covering a wide range of information from users: Flooding, GeoDest and GeoTrace, and have shown that our proposed protocol performs better or equally well than those while affording better privacy or lesser overheads.

\section{REFERENCES}

[1] C.E. Perkins and E.M. Royer, Ad-hoc on demand distance vector routing, in $2^{\text {nd }}$ IEEE Workshop on Mobile Computing Systems and Applications, pp. 90-100, Feb. 1999

[2] V. Namboodiri, M. Agarwal, and L. Gao, A study on the feasibility of mobile gateways for vehicular ad-hoc networks, in $1^{\text {st }}$ International Workshop on Vehicular Ad Hoc Networks, pp. 66-75, 2004.

[3] B. Karp and H. T. Kung. GPSR: greedy perimeter stateless routing for wireless networks, in Mobile Computing and Networking, 2000.

[4] H. Füßler, M. Mauve, H. Hartenstein, M. Kasemann, and D. Vollmer, Location based routing for vehicular ad-hoc networks, in ACM SIGMOBILE MC2R, vol. 7, no. 1, pp. 47-49, Jan. 2003

[5] C. Lochert, H. Hartenstein, J. Tian, D. Herrmann, H. Füßler, and M. Mauve, A routing strategy for vehicular ad hoc networks in city environments, in IV2003, June 2003.

[6] C. Lochert, M. Mauve, H. F“ussler, and H. Hartenstein. Geographic routing in city scenarios, In SIGMOBILE MC2R, 9(1):69-72, 2005.

[7] P.-C Cheng, J.-T. Weng, L.-C. Tung, K. C. Lee, M. Gerla, and J. Härri, GeoDTN+NAV: A Hybrid Geographic and DTN Routing with Navigation Assistance in Urban Vehicular Networks, in ISVCS, 2008.

[8] B. -C. Seet, G. Liu, B.-S. Lee, C.-H. Foh, K.-J. Wong, and K.-K. Lee, A-star: A mobile ad hoc routing strategy for metropolis vehicular communications, in NETWORKING 2004

[9] F. Giudici and E. Pagani, Spatial and traffic-aware routing (star) for vehicular systems, in High Performance Computing and Communications, 2005

[10] S. Jain, K. Fall, and R. Patra. Routing in a delay tolerant network. in SIGCOMM, 2004.

[11] I. Leontiadis and C. Mascolo. GeOpps: Geographical opportunistic routing for vehicular networks. World of Wireless, Mobile and Multimedia Networks, in WoWMoM, 2007.

[12] F. Xu, S. Guo, J. Jeong, Y. Gu, Q. Cao, M. Liu and T. He, Utilizing shared vehicle trajectories for data forwarding in vehicular networks, in INFOCOM, 2011

[13] M. Rondinone and J.Gozalvez, Exploiting Multi-hop Connectivity for Dynamic Routing in VANETs, in ISWCS, 2011.

[14] J. Tian, L. Han, K. Rothermel, and C. Cseh, Spatially aware packet routing for mobile ad hoc intervehicle radio networks, in Intelligent Transportation Systems, 2003.

[15] M. Jerbi, R. Meraihi, S.-M. Senouci, and Y. G.-Doudane, Gytar: Improved greedy traffic aware routing protocol for vehicular ad hoc networks in city environments, in VANET, 2006.

[16] J. Zhao, and G. Cao, VADD: Vehicle-Assisted Data Delivery in Vehicular Ad Hoc Networks, in INFOCOM 2006. 